\title{
RIGID COHOMOLOGY OF TOPOLOGICAL GROUPOIDS
}

\author{
K. A. MACKENZIE
}

(Received 15 April 1977)

Communicated by $\mathbf{J}$ Virsik

\begin{abstract}
A cohomology theory for locally trivial, locally compact topological groupoids with coefficients in vector bundles is constructed, generalizing constructions of Hochschild and Mostow (1962) for topological groups and Higgins (1971) for discrete groupoids. It is calculated to be naturally isomorphic to the cohomology of the vertex groups, and is thus independent of the twistedness of the groupoid. The second cohomology space is accordingly realized as those "rigid" extensions which essentially arise from extensions of the vertex group; the cohomological machinery now yields the unexpected result that in fact all extensions, satisfying some natural weak conditions, are rigid.
\end{abstract}

Subject classification (Amer. Math. Soc. (MOS) 1970): 18 H 40, 20 L 05.

\section{Introduction}

There already exist cohomology theories for discrete groupoids (Higgins (1971)) and for topological groups (Hochschild and Mostow (1962)). In this paper we construct the natural common generalization of these two theories, giving a cohomology theory for locally compact, locally trivial topological groupoids, and study the extension theory of these groupoids.

We have called this the "rigid" cohomology on account of the nature of the extensions classified by the second cohomology space: these extensions $E \succ \Phi \rightarrow \Omega$ are rigid in the sense that $\Phi$ is twisted exactly as $\Omega$ is ( $\Phi$ and $\Omega$ have the same local triviality properties). Since a locally trivial topological groupoid is determined by its vertex groups and its twistedness, one expects that rigid extensions will correspond to extensions of the vertex groups and this turns out to be so (7.4). In fact we prove (Theorem 3 ) that $H^{*}(\Omega, E)$ is naturally isomorphic in all degrees to the continuous cohomology of any vertex group. Although this is a natural result for 
trivial groupoids (in particular for discrete groupoids (Higgins (1971)), it is surprising that it holds in general for arbitrarily twisted groupoids. What is more surprising is that all extensions, satisfying some mild conditions, are in fact rigid. This result (Theorem 4) resists a direct proof, but the cohomological apparatus developed here allows it to be reduced to the semi-direct case, where it follows from a version of the result that principal bundles with vector groups are trivial.

We give no applications beyond this at present, but in a future paper we will construct an analogous cohomology for locally trivial Lie groupoids and relate it to the cohomology of their Lie algebroids, obtaining a van Est theorem for locally trivial Lie groupoids and a new approach, in this case, to the theorem of Pradines (1968).

The theory developed here is related to, but independent of, the cohomology sketched by Bott (1975) in a report only available to the author after this manuscript had been prepared. This paper gives full details, realizing the $H^{n}(\Omega, E)$ as derived functors on a category of coefficient modules, and studies the extension theory. For an account of continuous cohomology from the classifying space approach, see the forthcoming survey article by Stasheff (1978). The author is grateful to a referee for referring him to Professor Stasheff for this reference.

The newly revised theory of Moore (1976) clearly should extend to groupoids and generalize the present theory, but in the application to Lie groupoids and the van Est theory it is the Hochschild-Mostow approach which is necessary, and so we do not consider Moore's theory here.

\section{Topological groupoids}

Topological groupoids were introduced by Ehresmann (1959). Accounts of their elementary properties already exist in, for example, Brown and Hardy (1976) and the references given there. We therefore only establish our notations and conventions and record some observations need in the sequel.

Definition 1.1. Let $B$ be a set. A groupoid with base $B$ (or on $B$ ) is a small category $\Omega$ in which every element (morphism) is invertible, and whose set of objects is identified with $B$. For $x \in B$ we denote by $\tilde{x}$ the corresponding identity. The source and target maps $\Omega \rightarrow B$ are denoted by $a$ and $b$ respectively and

$$
\Omega * \Omega=\{(\xi, \eta) \in \Omega \times \Omega \mid a \xi=b \eta\}
$$

is the set of composable pairs. For $U, V \subset B$ we write $\Omega_{U}=a^{-1} U, \Omega^{V}=b^{-1} V$ and $\Omega_{U}^{V}=\Omega_{U} \cap \Omega^{V}$. In particular, $\Omega_{x}^{x}$ is the vertex group at $x \in B$. Lastly, $\Omega$ is transitive if $\Omega_{x}^{y}$ is nonvoid for all $x, y \in B$. 
DEFINITION 1.2. If $\Omega$ and $\Omega^{\prime}$ are groupoids with base $B$ then a morphism from $\Omega$ to $\Omega^{\prime}$ is a map $\varphi: \Omega \rightarrow \Omega^{\prime}$ such that $\varphi(\tilde{x})=\tilde{x}$ for all $x \in B, a^{\prime} \circ \varphi=a$ and $b^{\prime} \circ \varphi=b$, and $\varphi(\xi . \eta)=\varphi(\xi) . \varphi(\eta)$ for $\xi, \eta \in \Omega$.

Definition 1.3. Let $B$ be a Hausdorff space. A topological groupoid on $B$ is a transitive groupoid $\Omega$ with base $B$ equipped with a Hausdorff topology making $a$ and $b$, the multiplication $\Omega * \Omega \rightarrow \Omega$, and the inversion $\xi+\xi^{-1}$, continuous and the identification $x+\rightarrow \tilde{x}, B \rightarrow \Omega$ a homeomorphism into. ( $\Omega * \Omega$ has the pull-back topology.)

A morphism of topological groupoids is a morphism of the underlying groupoids which is continuous.

If $B$ is a space and $G$ a topological group (both Hausdorff) then $B \times G \times B$ with the product topology and groupoid structure $a(y, g, x)=x, b(y, g, x)=y$ and $(z, h, y) \cdot(y, g, x)=(z, h g, x)$ becomes a topological groupoid, called a product topological groupoid. In the algebraic theory, all groupoids are isomorphic to product groupoids; the interest of the topological theory is that this is not so.

DEFINITION 1.4. The topological groupoid $\Omega$ on base $B$ is locally trivial if the map $[b, a]: \Omega \rightarrow B \times B, \xi+(b \xi, a \xi)$ is a topological submersion (that is, it admits local right-inverses at each point of its range (Hardy (1971))). Equivalently, $\Omega$ is locally trivial if there exists a $w \in B$, an open cover $\left\{U_{\alpha}\right\}$ of $B$ and continuous maps $\sigma_{\alpha}: U_{\alpha} \rightarrow \Omega_{w}$ such that $\sigma_{\alpha}(x) \in \Omega_{w}^{x}$ for $x \in U_{\alpha}$ and $\sigma_{\alpha}(w)=\tilde{w}$ whenever $w \in U_{\alpha}$.

$\left\{U_{\alpha}\right\}$ is then said to be a decomposing cover for $\Omega$, and is said to decompose $\Omega$ through the family $\left\{\sigma_{\alpha}\right\}$. The maps $s_{\alpha \beta}: U_{\alpha \beta}=U_{\alpha} \cap U_{\beta} \rightarrow \Omega_{w}^{w}, x \mapsto \sigma_{\alpha}(x)^{-1} . \sigma_{\beta}(x)$ are the transition functions for $\Omega$ with respect to $\left\{\sigma_{\alpha}\right\}$.

Using $\left\{\sigma_{\alpha}\right\}$ we define decomposing homeomorphisms $h_{\alpha}: U_{\alpha} \times \Omega_{w} \rightarrow \Omega_{U_{\alpha}}$ by $(x, \zeta) \mapsto \zeta \sigma_{\alpha}(x)^{-1}$ with restriction $h_{a, x}: \Omega_{w} \rightarrow \Omega_{x}$ and $\Sigma_{\alpha}: U_{\alpha} \times \Omega_{w}^{w} \times U_{\alpha} \rightarrow \Omega_{U_{\alpha}^{\alpha}}^{U}$ by $(y, \lambda, x)+\sigma_{\alpha}(y) \cdot \lambda \cdot \sigma_{\alpha}(x)^{-1}$.

$\Sigma_{\alpha}$ is a (topological) isomorphism from the product groupoid $U_{\alpha} \times \Omega_{w}^{w} \times U_{\alpha}$ onto $\Omega_{U_{\alpha}^{\alpha}}^{U_{\alpha}}$, the restriction of $\Omega$ to $U_{\alpha}$. Since any topological isomorphism $\Omega_{U}^{U} \rightarrow U \times \Omega_{w}^{w} \times U$ defines a continuous $\sigma_{U}: U \rightarrow \Omega_{w}^{U}$, we take the view that the decomposing covers of $B$ give a measure of the 'twistedness' of $\Omega$, that is, of how far it departs from being globally isomorphic to a product groupoid.

Proposition 1.5. If $\varphi: \Omega \rightarrow \Omega^{\prime}$ is a morphism of topological groupoids and $\Omega$ is locally trivial with $\left\{U_{\alpha}\right\}$ a decomposing cover then $\Omega^{\prime}$ is also locally trivial and decomposed by $\left\{U_{\alpha}\right\}$. 
PROOF. If $\sigma_{\alpha}: U_{a} \rightarrow \Omega_{w}^{U_{\alpha}}$ are continuous then clearly $\varphi_{\circ} \sigma_{\alpha}: U_{\alpha} \rightarrow \Omega_{w}^{\prime} U_{\alpha}$ are also.

Thus "morphisms reduce twistedness" and in the category of locally trivial topological groupoids on a given base $B$, morphisms only exist between groupoids with compatible twistedness.

DEFINITION 1.6. A topological groupoid $\Omega$ on base $B$ is locally compact if the space $\Omega$ is locally compact.

It follows then that all $\Omega_{x}$ and $\Omega_{x}^{x}$ are locally compact.

For $X$ and $Y$ any topological spaces, $C(X, Y)$ denotes the space of all continuous maps $X \rightarrow Y$ with the compact-open topology. As is well known this topology only behaves well for locally compact $X$ and for this reason we have to restrict attention to locally compact groupoids.

\section{Continuous vector bundles}

The category of vector bundles of finite rank is not large enough to accommodate the injective resolutions of Section 3. We therefore introduce the natural generalization in which the fibres may be arbitrary locally convex spaces. That this is the appropriate concept is shown by Proposition 2.3 and Theorem 3 of Section 7.

Throughout the rest of the paper, $B$ denotes a paracompact and connected $C^{0}$ manifold. A Hausdorff locally convex $\mathbf{R}$-vector space is referred to as an LCS. For the properties of LCS's which we use, see Choquet (1969). Recall that an LCS is locally compact if and only if it is finite dimensional.

Definition 2.1. A continuous vector bundle (CVB) on $B$ is a triple $(E, p, B)$ in which $E$ is a space and $p$ a continuous onto map with $E_{x}=p^{-1}(x)$ an LCS for all $x \in B$ and the local triviality property: there exists an open cover $\left\{U_{\alpha}\right\}$ of $B$, an LCS $A$ and decomposing maps $\psi_{\alpha}$ which are homeomorphisms $U_{\alpha} \times A \rightarrow E_{U_{\alpha}}=p^{-1}\left(U_{\alpha}\right)$ such that $p \circ \psi_{\alpha}=\pi_{1}: U_{\alpha} \times A \rightarrow U_{\alpha}$ and the restrictions $\psi_{\alpha, x}: A \rightarrow E_{x}, a+\psi_{\alpha}(x, a)$ are linear homeomorphisms.

For CVB's $E$ and $E^{\prime}$ on $B$ a $C V B$ morphism is a continuous, fibre-preserving, fibrewise linear map $\varphi: E \rightarrow E^{\prime}$.

REMARK. Vector bundles, and their morphisms, in the sense of Lang (1975) are clearly particular cases of (2.1).

For an LCS $A$, we prefer to leave the set of linear homeomorphisms $A \rightarrow A$ without a topology and regard the transition functions as continuous maps $U_{\alpha \beta} \times A \rightarrow A$. The following construction principle can be easily provided. 
Proposition 2.2. If $B$ is a $C^{0}$ manifold, paracompact and connected, $A$ is an LCS and $E_{x}$ is an LCS for each $x \in B, E$ is the disjoint union of the $E_{x}, x \in B$ and $p: E \rightarrow B$ is the obvious projection, then if there are linear homeomorphisms $\psi_{\alpha, x}: A \rightarrow E_{N}$, $x \in U_{\alpha}$ for all $U_{\alpha}$ in an open cover of $B$ such that the transition functions $U_{\alpha \beta} \times A \rightarrow A$ by $(x, a) \rightarrow \psi_{\alpha, x}^{-1} \circ \psi_{\beta, x}(a)$ are continuous, then there is a unique topology on $E$ which makes $(E, p, B)$ into a CVB with decompositions $\psi_{\alpha}: U_{\alpha} \times A \rightarrow E_{U_{\alpha}},(x, a)+\psi_{\alpha, x}(a)$.

Thus the usual functorial constructions (direct sums, bundles of linear maps and so forth) can be carried out with CVB's. The following construction will be needed in the sequel.

(2.3). Let $\Omega$ be a locally trivial, locally compact topological groupoid on $B$, and $(E, p, B)$ a CVB on $B$. For $x \in B, C\left(\Omega_{x}, E_{x}\right)$ denotes the LCS of all continuous maps $\Omega_{x} \rightarrow E_{x}$ with the compact-open topology. We construct a natural CVB $F(\Omega, E)$ on $B$ with $F(\Omega, E)_{x}=C\left(\Omega_{x}, E_{x}\right)$ for $x \in B$.

Let $\left\{U_{\alpha}\right\}$ be an open cover of $B$ which decomposes $\Omega$ through $\sigma_{\alpha}: U_{\alpha} \rightarrow \Omega_{w}$ and $E$ through $\psi_{\alpha}: U_{\alpha} \times A \rightarrow E_{U_{\alpha}}$. Write $P=\Omega_{w}$ and $h_{\alpha}: U_{\alpha} \times P \rightarrow \Omega_{U_{\alpha}}$ for the map $(x, \zeta) \rightarrow \zeta . \sigma_{\alpha}(x)^{-1}$. Then $b \circ h_{\alpha}=b \circ \pi_{2}\left(\pi_{2}: U_{\alpha} \times P \rightarrow P\right)$ and $h_{\alpha}(x, \eta \zeta)=\eta \cdot h_{\alpha}(x, \zeta)$. Define

$$
\bar{\psi}_{\alpha x}: C(P, A) \rightarrow C\left(\Omega_{x}, E_{x}\right) \quad\left(x \in U_{\alpha}\right)
$$

by

$$
f \nrightarrow \psi_{\alpha, x} \circ f \circ h_{\alpha, x}^{-1}
$$

Then $\bar{\psi}_{\alpha, x}$ is clearly linear; to prove its continuity we consider the associate map $C(P, A) \times \Omega_{x} \rightarrow E_{x}$, which is the composite of $\psi_{\alpha, x}: A \rightarrow E_{x}$ and $C(P, A) \times \Omega_{x} \rightarrow A$ by $(f, \xi)+f\left(h_{\alpha, x}^{-1}(\xi)\right)$ which is continuous because it is

$$
\text { id } \times h_{\alpha, x}^{-1}: C(P, A) \times \Omega_{x} \rightarrow C(P, A) \times P
$$

followed by the evaluation map $C(P, A) \times P \rightarrow A$ of the locally compact $P$. Similarly $\psi_{\alpha, x}^{-1}$ is continuous and so $\psi_{\alpha, x}$ is a linear homeomorphism.

The transition function $U_{\alpha \beta} \times C(P, A) \rightarrow C(P, A)$ has associate

$$
U_{\alpha \beta} \times C(P, A) \times P \rightarrow A
$$

by

$$
(x, f, \zeta) \rightarrow \pi_{2}^{A} \circ \psi_{\alpha}^{-1} \circ \psi_{\beta}\left(x, f\left(\pi_{2}^{P} \circ h_{\beta}^{-1} \circ h_{\alpha}(x, \zeta)\right)\right)
$$

where $\pi_{2}^{A}: U_{\alpha \beta} \times A \rightarrow A$ and $\pi_{2}^{P}: U_{\alpha \beta} \times P \rightarrow P$ are the projections, and the continuity of this follows from that of $\pi_{2}^{A} \circ \psi_{\alpha}^{-1} \circ \psi_{\beta}: U_{\alpha \beta} \times A \rightarrow A$ and $\pi_{2}^{P} \circ h_{\beta}^{-1} \circ h_{\alpha}: U_{\alpha \beta} \times P \rightarrow P$ and of the evaluation map $C(P, A) \times P \rightarrow A$ of the locally compact $P$. 
So by Proposition 2.2 we have proved:

Proposition 2.3. Given a locally trivial, locally compact topological groupoid $\Omega$ and $a C V B E$, both on base $B$, there is a unique $C V B F(\Omega, E)$ on $B$ with $F(\Omega, E)_{x}=C\left(\Omega_{x}, E_{x}\right)$ and such that if $\left\{U_{\alpha}\right\}$ decomposes both $\Omega$ and $E$ then it also decomposes $F(\Omega, E)$ as described above.

We complete this section with the following description of the topology for $\Gamma_{U} E$, the space of continuous local sections of $E$ over $U$.

Proposition 2.4. Let $(E, p, B)$ be $a C V B$ on $B$ and $U \subset B$ an open set. If $\left\{U_{\alpha}\right\}$ is a finite decomposing cover for $E_{U}$ with decomposing homeomorphisms $\psi_{\alpha}: U_{\alpha} \times A \rightarrow E_{U_{\alpha}}$ and $\left\{p_{j}\right\}$ is a family of semi-norms on the LCS $A$ which define its topology, define $p_{j}^{\alpha, K}: \Gamma_{U} E \rightarrow \mathbf{R}^{+}$by $p_{j}^{\alpha, K}(\sigma)=\sup \left\{p_{j}\left(\psi_{\alpha, x}^{-1}(\sigma(x))\right) \mid x \in K\right\}, \quad \sigma \in \Gamma_{U} E$ for compact $K \subset U_{\alpha}$ and any $j, \alpha$. Then the $\left\{p_{j}^{\alpha, K}\right\}$ define the compact-open topology on $\Gamma_{U} E$, which therefore makes $\Gamma_{U} E$ an LCS.

\section{The category of continuous $\Omega$-modules}

In this and the next two sections we construct the rigid cohomology of a locally trivial, locally compact topological groupoid as the derived functors of a natural fixed-point functor. We follow the techniques of Hochschild and Mostow (1962) in general, but the nature of groupoids forces some changes which make the details more difficult. (This becomes more apparent in the Lie case.)

Throughout Sections $3-5, \Omega$ is a locally trivial, locally compact topological groupoid with base $B$.

Definition 3.1 (Ehresmann (1959)). A continuous $\Omega$-module is a CVB $(E, p, B)$ together with a continuous action $\Omega * E \rightarrow E,(\xi, u)+\xi . u$ of $\Omega$ on $E$. Here

$$
\Omega * E=\{(\xi, u) \mid a \xi=p u\} \subset \Omega \times E
$$

has the pullback topology, and that $\Omega * E \rightarrow E$ is an action means that

(i) $p(\xi, u)=b \xi$ for all $(\xi, u) \in \Omega * E$,

(ii) $\xi: E_{a \xi} \rightarrow E_{b \xi}, u \mapsto \rightarrow \xi . u$ is a linear homeomorphism,

(iii) $\eta \cdot(\xi \cdot u)=(\eta \cdot \xi) \cdot u$ whenever either side is defined,

(iv) $\widetilde{p u} \cdot u=u$ for all $u \in E$.

It was proved by Seda (1975) that if a vertex group $\Omega_{x}^{x}$ acts on a vector space $V$ linearly, then there is a vector bundle on $B$ with fibre type $V$ on which $\Omega$ acts continuously. Thus there are always many continuous $\Omega$-modules. 
It can be proved that if $E$ is a continuous $\Omega$-module then the associate map $\Omega \rightarrow \Pi(E)$ is a continuous morphism where $\Pi(E)$ is the topological groupoid of isomorphisms between fibres of $E$ with the compact-open topology on each $\Pi(E)_{x}^{y}$.

Definition 3.2. If $E$ and $E^{\prime}$ are continuous $\Omega$-modules, then a continuous $\Omega$-module morphism $\varphi: E \rightarrow E^{\prime}$ is a CVB morphism for which $\varphi(\xi . u)=\xi . \varphi(u)$ for all $(\xi, u) \in \Omega * E$.

The set of all continuous $\Omega$-module morphisms $E \rightarrow E^{\prime}$ forms an $\mathbf{R}$-vector space $\operatorname{Hom}_{\Omega}\left(E, E^{\prime}\right)$. We will not need to consider a vector topology on $\operatorname{Hom}_{\Omega}\left(E, E^{\prime}\right)$ in general and so it remains a vector space without topology.

Thus we have the category of continuous $\Omega$-modules and their morphisms, denoted $\Omega$-Mod. It is easily seen to be an abelian category (note that kernels and quotients exist because continuous $\Omega$-module morphisms are automatically of constant rank). Like Hochschild and Mostow, we will actually be deriving functors relative to the class of exact sequences in $\Omega$-Mod which split in the CVB category, but we will not use the terminology of relative homological algebra.

Definirion 3.3. A sequence $E \stackrel{\varphi}{\longrightarrow} E^{\prime} \stackrel{\psi}{\longrightarrow} E^{\prime \prime}$ of continuous $\Omega$-module morphisms is exact at $E^{\prime}$ if it is exact as a sequence of CVB morphisms in the usual sense and there are CVB morphisms $\lambda: E^{\prime} \rightarrow E$ and $\rho: E^{\prime \prime} \rightarrow E^{\prime}$ such that $\varphi \circ \lambda+\rho \circ \psi=\mathrm{id}_{E^{\prime}}$ and $\lambda \circ \rho=0: E^{\prime \prime} \rightarrow E$.

An $\Omega$-monomorphism, denoted $\varphi: E \nrightarrow E^{\prime}$, and also called a continuous imbedding of $E$ into $E^{\prime}$ is a continuous $\Omega$-module morphism $\varphi$ such that $0 \rightarrow E \stackrel{\varphi}{\longrightarrow} E^{\prime}$ is exact at $E$.

An $\Omega$-epimorphism, denoted $\varphi: E \rightarrow E^{\prime}$ is a continuous $\Omega$-module morphism $\varphi$ such that $E \stackrel{\varphi}{\longrightarrow} E^{\prime} \longrightarrow 0$ is exact at $E^{\prime}$.

Thus a $1: 1$ continuous $\Omega$-module morphism $\varphi: E \rightarrow E^{\prime}$ is an $\Omega$-monomorphism if and only if there is a CVB morphism $\lambda: E^{\prime} \rightarrow E$ such that $\lambda \circ \varphi=\mathrm{id}_{E}$. If $E$ and $E^{\prime}$ are of finite rank then $\lambda$ exists automatically. The dual statements for $\Omega$-epimorphisms also hold.

Definition 3.4. A continuous $\Omega$-module $E$ is continuously injective if, given any continuous $\Omega$-module morphism $\varphi: E^{\prime} \rightarrow E$ and an $\Omega$-monomorphism $\psi: E^{\prime} \rightarrow E^{\prime \prime}$, there exists a lifting, that is, a continuous $\Omega$-module morphism $\tilde{\varphi}: E^{\prime \prime} \rightarrow E$ such that $\tilde{\varphi} \circ \psi=\varphi$.

Consider now one continuous $\Omega$-module $E$ until the end of Section 3 . We constructed in (2.3) the CVB $F(\Omega, E)$ on $B$ and we now make it into a continuous 
$\Omega$-module by defining

(3.5) $(\xi . f)(\eta)=\xi \cdot f(\eta \xi)$

for $\xi \in \Omega_{x}^{\nu}, f \in F(\Omega, E)_{x}, \eta \in \Omega_{y}$ and any $x, y \in B$.

Proposition 3.6. $F(\Omega, E)$ is a continuous $\Omega$-module under the action (3.5).

Proof. The algebraic properties are easily verified. It remains to show the continuity of $\Omega^{*} F(\Omega, E) \rightarrow F(\Omega, E)$. Using the notation of (2.3), it suffices to prove that

$$
\begin{aligned}
\Omega_{U_{\alpha}}^{U^{\beta}} *\left(U_{\alpha} \times C(P, A)\right) & \rightarrow U_{\beta} \times C(P, A), \\
(\xi, a \xi, f) & \rightarrow \psi_{\beta}^{-1}\left(\xi . \psi_{a}(a \xi, f)\right)
\end{aligned}
$$

is continuous, and this reduces as before to the associate map, and so since $\pi_{2} \circ \psi_{\beta}^{-1}$ is continuous we need only show that

$$
\begin{gathered}
\Omega_{U_{x}^{B} \times C(P, A) \times P \rightarrow E_{U_{\beta}}^{U_{\beta}},} \\
(\xi, f, \zeta)+\xi \cdot \psi_{\alpha, a \xi}\left(f\left(h_{\beta}(b \xi, \zeta) \cdot h_{\alpha, a \xi}^{-1}(\xi)\right)\right)
\end{gathered}
$$

is continuous. This is the composite of

$$
\begin{gathered}
\Omega_{U_{\alpha}^{\beta} \times C(P, A) \times P \rightarrow \Omega_{U_{\alpha}^{\beta}}^{U^{\beta}} \times P \times C(P, A),} \\
(\xi, f, \zeta)+\left(\xi, h_{\rho}(b \xi, \zeta) .\left(\pi_{2} \circ h_{\alpha}^{-1}(\xi)\right), f\right),
\end{gathered}
$$

which is continuous since the operations in $\Omega$ are continuous,

$$
\text { id } \times \text { ev: } \Omega_{U_{x}^{B}}^{U} \times P \times C(P, A) \rightarrow \Omega_{U_{x}^{B}}^{U} \times A,
$$

which is continuous since $P$ is locally compact, and

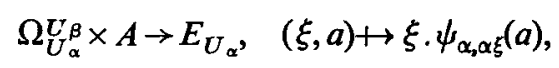

which is continuous because $E$ is itself a continuous $\Omega$-module. Thus the continuity of $\Omega^{*} E \rightarrow E$ is lifted to show the continuity of $\Omega^{*} F(\Omega, E) \rightarrow F(\Omega, E)$ and this completes the proof.

Proposition 3.7. $F(\Omega, E)$ is a continuously injective $\Omega$-module.

Proof. Let

$$
\begin{aligned}
& \psi E^{\prime \prime} \lambda \lambda \\
& E^{\prime} \stackrel{\varphi}{\longrightarrow} F(\Omega, E)
\end{aligned}
$$


be given with $\lambda$ a CVB morphism such that $\lambda_{\circ} \psi=\mathrm{id}_{E^{\prime}}$. Define $\tilde{\varphi}: E^{\prime \prime} \rightarrow F(\Omega, E)$ by $\tilde{\varphi}(v)(\xi)=\xi^{-1}(\varphi(\lambda(\xi . v))(\widetilde{b \xi}))$ for $\xi \in \Omega_{x}, v \in E_{x}^{\prime \prime}$ and $x \in B$. $\tilde{\varphi}$ can be shown to be a CVB morphism by standard techniques, and is easily verified to be an $\Omega$-morphism. Lastly,

$$
\begin{aligned}
(\tilde{\varphi} \circ \psi)(u)(\xi) & =\xi^{-1} \cdot(\varphi(\lambda \circ \psi(\xi \cdot u))(\widetilde{b \xi}))=\xi^{-1} \cdot(\varphi(\xi \cdot u)(\widetilde{b \xi})) \\
& =\xi^{-1} \cdot \xi \cdot \varphi(u)(\widetilde{b \xi} \cdot \xi)=\varphi(u)(\xi)
\end{aligned}
$$

for all $u \in E_{x}^{\prime}, \xi \in \Omega_{x}$ and $x \in B$. So $\tilde{\varphi} \circ \psi=\varphi$ as required.

Proposition 3.8. The constant map $\kappa: E \rightarrow F(\Omega, E)$, defined by $\kappa(u)(\xi)=u$ for $u \in E_{x}, \xi \in \Omega_{x}, x \in B$, is an $\Omega$-monomorphism.

PROOF. That $\kappa$ is a CVB morphism is obvious; for $u \in E_{x}, \xi \in \Omega_{x}^{y}$ and $\eta \in \Omega_{y}$ we have $(\xi . \kappa(u))(\eta)=\xi \cdot(\kappa(u)(\eta \xi))=\xi \cdot u=\kappa(\xi . u)(\eta)$ and so $\kappa$ is a continuous $\Omega$-module morphism. It is clearly $1: 1$ and $\lambda: F(\Omega, E) \rightarrow E, f+f(\widetilde{p(f)})$ is a CVB morphism and a left inverse for $\kappa$.

We have thus proved:

THEOREM 1. A continuous $\Omega$-module can be continuously imbedded in a continuously injective $\Omega$-module. In particular, $\Omega-M o d$ has sufficiently many injectives.

With this result we can apply the standard techniques of homological algebra in the category $\Omega$-Mod.

Remark. It is clear when $B$ is a point, so that $\Omega$ is in fact a locally compact group, that the category of continuous $\Omega$-modules coincides with that of Hochschild and Mostow (1962). For a continuous $\Omega$-module $E$, the CVB (that is, LCS) $F(\Omega, E)$ coincides with that defined by Hochschild and Mostow, and the $\Omega$-module structure (3.5) is equivalent to the second action defined by Hochschild and Mostow under the automorphism $f \rightarrow f^{\prime}$ where $f^{\prime}(\xi)=f\left(\xi^{-1}\right)$ for all $\xi \in \Omega$. (This equivalence is a CVB morphism only when $B$ is a point.) For general groupoids, there is no analogue of Hochschild and Mostow's first and simpler action, and this is the source of some technical complications, especially in the Lie case.

\section{The fixed-point functor}

The product CVB $B \times \mathbf{R} \rightarrow B$ is a continuous $\Omega$-module under the trivial action $\xi .(x, t)=(y, t)$ for $\xi \in \Omega_{x}^{y}, t \in \mathbf{R}$, and we consider the fixed-point functor $\operatorname{Hom}_{\Omega}(B \times \mathbf{R},-)$ from $\Omega$-Mod to, at present, the category of $\mathbf{R}$-vector spaces and 
write

$$
H^{0}(\Omega, E)=E^{\Omega}=\operatorname{Hom}_{\Omega}(B \times \mathbf{R}, E) \text { and } \theta_{*}=\operatorname{Hom}_{\Omega}(B \times \mathbf{R}, \theta)
$$

for objects $E$ and morphisms $\theta$ in $\Omega$-Mod.

Elements of $\operatorname{Hom}_{\Omega}(B \times \mathbf{R}, E)$ are CVB morphisms $\varphi: B \times \mathbf{R} \rightarrow E$ such that $\varphi(y, t)=\xi \cdot \varphi(x, t)$ for all $\xi \in \Omega_{x}^{y}, t \in \mathbf{R}$ and $x, y \in B$. If $\varphi$ is restricted to $\{n \cdot\} \times \mathbf{R} \rightarrow E_{w}$ for some fixed $w \in B$, we get an element of $\operatorname{Hom}_{G}(\mathbf{R}, A)$ where $G=\Omega_{w}^{w}$ and $A=E_{w}$. On the other hand, $x+\varphi(x, 1)$ defines a section $\sigma$ of $E$ which is invariant in the sense that $\xi . \sigma(a \xi)=\sigma(b \xi)$ for all $\xi \in \Omega$. There are thus maps

$$
\operatorname{Hom}_{\Omega}(B \times \mathbf{R}, E) \rightarrow \operatorname{Hom}_{G}(\mathbf{R}, A), \quad \operatorname{Hom}_{\Omega}(B \times \mathbf{R}, E) \rightarrow(\Gamma E)^{\Omega},
$$

when $(\Gamma E)^{\Omega}$ denotes the invariant sections of $E$, and by defining

$$
\begin{aligned}
\operatorname{Hom}_{G}(\mathbf{R}, A) \rightarrow A^{G}, & f \leftrightarrow f(1), \\
(\Gamma E)^{\Omega} \rightarrow A^{G}, & \sigma \mapsto \sigma(w),
\end{aligned}
$$

we get a commutative diagram

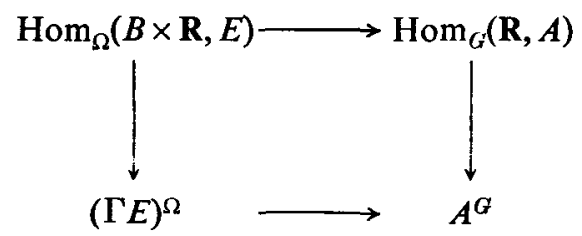

in which each side is a linear isomorphism. Thus $\sigma \in(\Gamma E)^{\Omega}$ defines

$$
\varphi \in \operatorname{Hom}_{\Omega 2}(B \times \mathbf{R}, E)
$$

by $\varphi(x, t)=t \cdot \sigma(x)$ and $f \in \operatorname{Hom}_{G}(\mathbf{R}, A)$ defines a $\varphi$ in $\operatorname{Hom}_{\Omega}(B \times \mathbf{R}, E)$ by $\varphi(x, t)=\xi \cdot f(t)$ for any $\xi \in \Omega_{w}^{x}$. An element $u \in A^{G}$ defines a $\sigma \in(\Gamma E)^{\Omega}$ by $\sigma(x)=\xi \cdot u$ for any $\xi \in \Omega_{w}^{x}$, also well defined, and an $f \in \operatorname{Hom}_{G}(\mathbf{R}, A)$ by $f(t)=t$. $u$ for $t \in \mathbf{R}$.

Now $(\Gamma E)^{\Omega}, A^{G}$ and $\operatorname{Hom}_{G}(\mathbf{R}, A)$ are all LCS's under natural topologies: $(\Gamma E)^{\Omega}$ and $A^{G}$ as subspaces of $\Gamma E$ and $A$ respectively and $\operatorname{Hom}_{G}(\mathbf{R}, A)$ with the compactopen topology as a function space. It is easily verified that the two maps between these spaces are linear homeomorphisms, and so one can put a unique structure of LCS on $\operatorname{Hom}_{\Omega}(B \times \mathbf{R}, E)$ so that each side of (4.1) is a linear homeomorphism. This is most easily defined by the seminorms

$$
\bar{q}_{j}: \varphi \mapsto q_{j}(\varphi(w, 1)),
$$

where $\left\{q_{j}\right\}$ are the seminorms on $A$. 
It can now easily be verified that $\operatorname{Hom}_{\Omega}(B \times \mathbf{R},-)$ is a functor from $\Omega$-Mod to the category of LCS's.

Proposition 4.2. $\operatorname{Hom}_{\Omega}(B \times \mathbf{R},-)$ is left exact.

Proof. Let $0 \longrightarrow E \stackrel{\theta}{\longrightarrow} E^{\prime} \stackrel{\theta^{\prime}}{\longrightarrow} E^{\prime \prime}$ be an exact sequence in $\Omega$-Mod. Thus there are CVB morphisms $\lambda: E^{\prime} \rightarrow E$ and $\rho: E^{\prime \prime} \rightarrow E^{\prime}$ such that $\lambda_{\circ} \theta=\mathrm{id}_{E}$ and $\theta \circ \lambda+\rho \circ \theta^{\prime}=\mathrm{id}_{E^{\prime}}$. Consider

$$
0 \longrightarrow \operatorname{Hom}_{\Omega}(B \times \mathbf{R}, E) \stackrel{\theta_{*}}{\longrightarrow} \operatorname{Hom}_{\Omega}\left(B \times \mathbf{R}, E^{\prime}\right) \stackrel{\theta_{*}^{\prime}}{\longrightarrow} \operatorname{Hom}_{\Omega}\left(B \times \mathbf{R}, E^{\prime \prime}\right) .
$$

One proves easily that $\theta_{*}$ is $1: 1$ and that $\operatorname{im} \theta_{*} \subset \operatorname{ker} \theta_{*}^{\prime}$. If $\varphi \in \operatorname{Hom}_{\Omega}\left(B \times \mathbf{R}, E^{\prime}\right)$ is in $\operatorname{ker} \theta_{*}^{\prime}$ so that $\theta^{\prime} \circ \varphi=0$ then we can prove that $\lambda \circ \varphi \in \operatorname{Hom}_{\Omega}(B \times \mathbf{R}, E)$ by using the fact that $\theta *$ is $1: 1$ and noting that

$$
\left(\theta_{*}(\lambda \circ \varphi)\right)(y, t)=\varphi(y, t)-\rho . \theta^{\prime} \circ \varphi(y, t)=\varphi(y, t)
$$

whilst

$$
\theta_{*}(\xi \cdot(\lambda \circ \varphi))(x, t)=\xi \cdot \theta((\lambda \circ \varphi)(x, t))=\xi \cdot \varphi(x, t)=\varphi(y, t) \quad \text { for } \xi \in \Omega_{x}^{y}
$$

Hence $\operatorname{ker} \theta_{*}^{\prime} \subset \operatorname{im} \theta^{*}$. It appears not to be true that (4.3) is exact in the stronger sense of (3.3) (this already occurs for groups). However, a similar calculation shows that $\lambda_{w}: E_{w}^{\prime} \rightarrow E_{w}$ restricts to a continuous linear map $\operatorname{ker} \theta_{*}^{\prime} \rightarrow \operatorname{Hom}_{\Omega}(B \times \mathbf{R}, E)$ where $\operatorname{ker} \theta_{*}^{\prime}$ is identified with a subspace of $E_{w}^{\prime}$.

This justifies the notation $H^{0}(\Omega, E)=\operatorname{Hom}_{\Omega}(B \times \mathbf{R}, E)$; if

$$
0 \longrightarrow E \underset{\lambda^{0}}{\stackrel{c}{\rightleftarrows}} I^{0} \underset{\lambda^{1}}{\stackrel{d^{0}}{\rightleftarrows}} I^{1} \underset{\lambda^{2}}{\stackrel{d^{1}}{\rightleftarrows}} I^{2} \underset{\lambda^{3}}{\stackrel{d^{2}}{\rightleftarrows}} \ldots
$$

is a continuously injective resolution of $E$ in $\Omega-\mathrm{Mod}$, then $\operatorname{ker} d_{*}^{0}=\operatorname{im} c_{*}$ is topologically and linearly isomorphic to $\operatorname{Hom}_{\Omega}(B \times \mathbf{R}, E)$ under $c_{*}$ with inverse the restriction of $\lambda_{w}^{0}$.

For $n>0$,

$$
H^{n}(\Omega, E)=\frac{\operatorname{ker} d_{*}^{n}}{\operatorname{im} d_{*}^{n-1}}
$$

is the rigid cohomology of $\Omega$ with coefficients in the continuous $\Omega$-module $E$; standard arguments prove that the quotient is independent up to natural linear homeomorphisms, of the continuously injective resolution chosen (cf. Hochschild and Mostow (1962)). 
Similarly one proves that an exact sequence of continuous $\Omega$-modules $E \hookrightarrow E^{\prime} \rightarrow E^{\prime \prime}$ induces a long exact (in the weak sense of (4.2)) sequence in cohomology

$$
0 \longrightarrow H^{0}(\Omega, E) \longrightarrow H^{0}\left(\Omega, E^{\prime}\right) \longrightarrow H^{0}\left(\Omega, E^{\prime \prime}\right) \stackrel{\partial}{\longrightarrow} H^{1}(\Omega, E) \longrightarrow \ldots
$$

with the usual functorial properties, so that the $H^{n}(\Omega,-)$ form a connected sequence of functors.

Proposition 4.4. For $E$ a continuously injective $\Omega$-module, $H^{n}(\Omega, E)=(0)$ for all $n>0$.

Proof.

$$
0 \longrightarrow E \stackrel{\text { id }}{\longrightarrow} E \longrightarrow 0 \longrightarrow 0 \longrightarrow \ldots
$$

is a continuously injective resolution for $E$.

The diagram (4.1) can now be summarized in

Proposition 4.5. For any continuous $\Omega$-module $E$, and $w \in B$, there is a natural linear homeomorphism $H^{0}(\Omega, E) \cong H^{0}\left(\Omega_{w}^{w} . E_{w}\right)$.

When $B$ is a point, $\operatorname{Hom}_{\Omega}(B \times \mathbf{R},-)$ is the fixed-point functor considered by Hochschild and Mostow (1962). Therefore when $\Omega$ is a group, $H^{n}(\Omega, E)$ is the Hochschild-Mostow continuous cohomology of $\Omega$.

\section{The standard resolution}

Throughout Section $5, E$ is a fixed continuous $\Omega$-module. Define continuously injective $\Omega$-modules $F^{(n)}(\Omega, E)$ by

$$
F^{(0)}(\Omega, E)=F(\Omega, E), \quad F^{(n+1)}(\Omega, E)=F\left(\Omega, F^{(n)}(\Omega, E)\right), \quad n \geqslant 0 .
$$

We construct isomorphic copies of the $F^{(n)}(\Omega, E)$ which are easier to work with.

For each $x \in B$, let $F^{n}(\Omega, E)_{x}$ be $C\left(\Omega_{x}^{n+1}, E_{x}\right)$, the space of all continuous maps from the $(n+1)$-fold product of $\Omega_{x}$ to $E_{x}$ with the compact-open topology $(n \geqslant 0)$. We make

$$
F^{n}(\Omega, E)=\bigcup_{x \in B} F^{n}(\Omega, E)_{x}
$$

into a CVB in the obvious way by defining (notation of (2.3))

$$
\bar{\psi}_{x x}^{n}: F^{n}(P, A) \rightarrow F^{n}(\Omega, E)_{x}
$$


by $f+\rightarrow \psi_{\alpha, x} \circ f \circ\left(h_{\alpha x}^{-1}\right)^{n+1}$. If we define

$$
\begin{gathered}
\Omega * F^{n}(\Omega, E) \rightarrow F^{n}(\Omega, E) \\
(\xi, \varphi) \rightarrow\left(\left(\xi_{0}, \ldots, \xi_{n}\right) \rightarrow \xi \cdot \varphi\left(\xi_{0} \cdot \xi, \ldots, \xi_{n} \cdot \xi\right)\right)
\end{gathered}
$$

then we we can prove, as in (3.6), that $F^{n}(\Omega, E)$ is a continuous $\Omega$-module. Clearly $F^{0}(\Omega, E)=F(\Omega, E)$.

PROPOSITION 5.2. There are natural continuous $\Omega$-module isomorphisms

$$
I^{n}: F^{(n)}(\Omega, E) \rightarrow F^{n}(\Omega, E)
$$

with inverse

$$
\left(I^{n}\right)^{-1}(\varphi)\left(\xi_{0}\right)\left(\xi_{1}\right) \ldots\left(\xi_{n}\right)=\varphi\left(\xi_{0}, \ldots, \xi_{n}\right)
$$

ProOf. (Induction.) $I^{0}$ is the identity on $F(\Omega, E)$. Assume $I^{n}$ given and let $\tilde{I}^{n}: F^{(n+1)}(\Omega, E)=F\left(\Omega, F^{(n)}(\Omega, E)\right) \rightarrow F\left(\Omega, F^{n}(\Omega, E)\right)$ denote the induced isomorphism. We will prove that $\mathscr{A}: F\left(\Omega, F^{n}(\Omega, E)\right) \rightarrow F^{n+1}(\Omega, E)$ by

$$
\mathscr{A}(f)\left(\xi_{0}, \ldots, \xi_{n+1}\right)=f\left(\xi_{0}\right)\left(\xi_{1}, \ldots, \xi_{n+1}\right)
$$

is an isomorphism of continuous $\Omega$-modules, and then set $I^{n+1}=\mathscr{A} \circ I^{n}$. Since $\Omega_{x}$ is locally compact, each $\mathscr{A}_{x}: C\left(\Omega_{x}, C\left(\Omega_{x}^{n+1}, E_{x}\right)\right) \rightarrow C\left(\Omega_{x}^{n+2}, E_{x}\right)$ is a linear homeomorphism, and so, noting that $\psi_{\alpha}^{n+1} \circ\left(\mathrm{id}_{U_{\alpha}} \times \mathscr{A}_{w}\right)=\mathscr{A} \circ \bar{\psi}_{\alpha}$ as maps $U_{\alpha} \times C\left(P, F^{n}(P, A)\right) \rightarrow F^{n+1}(\Omega, E)_{U_{\alpha}}$ it follows that $\mathscr{A}$ is a CVB isomorphism $\left(\bar{\psi}_{\alpha}\right.$ is the chart on $\left.F\left(\Omega, F^{n}(\Omega, E)\right)\right)$. That $\mathscr{A}$ preserves the actions is easily verified, as is the last statement of the proposition.

Thus the $F^{n}(\Omega, E), n \geqslant 0$, are continuously injective $\Omega$-modules. We define a coboundary by the usual formula:

$$
\begin{gathered}
d^{n}: F^{n}(\Omega, E) \rightarrow F^{n+1}(\Omega, E) \\
d^{n}(f)\left(\xi_{0}, \ldots, \xi_{n+1}\right)=\sum_{j=0}^{n+1}(-1)^{j} f\left(\xi_{0}, \ldots, \xi_{j}, \ldots, \xi_{n+1}\right)
\end{gathered}
$$

and $d^{n}$ is easily seen to be a continuous $\Omega$-module morphism.

Proposition 5.4.

$$
0 \longrightarrow E \longrightarrow F^{0}(\Omega, E) \stackrel{d^{0}}{\longrightarrow} F^{1}(\Omega, E) \stackrel{d^{1}}{\longrightarrow}
$$

is a continuously injective resolution of $E$, called the standard resolution. 
Proof. It only remains to be shown that (5.4) is exact. It is easily seen that $\lambda^{n+1}: F^{n+1}(\Omega, E) \rightarrow F^{n}(\Omega, E)$ by $\lambda^{n+1}(f)\left(\xi_{0}, \ldots, \xi_{n}\right)=f\left(\tilde{x}, \xi_{0}, \ldots, \xi_{n}\right)$ for

$$
f \in F^{n+1}(\Omega, E)_{x}, \quad \xi_{j} \in \Omega_{x}, x \in B \text { and } \lambda^{0}: F^{0}(\Omega, E) \rightarrow E
$$

by $\lambda^{0}(f)=f(\tilde{x})$ for $f \in F(\Omega, E)_{x}, x \in B$, are CVB morphisms and satisfy $\lambda^{0} \circ \kappa=\mathrm{id}_{E}$, $\kappa \circ \lambda^{0}+\lambda^{1} \circ d^{0}=\mathrm{id}_{F(\Omega, E)}$, and $\lambda^{n+2} \circ d^{n+1}+d^{n} \circ \lambda^{n+1}=\mathrm{id}_{F^{n+1}(\Omega, E)}$. Thus (5.4) is exact.

Applying $\operatorname{Hom}_{\Omega}(B \times \mathbf{R},-)$ to (5.4) yields the homogeneous cochain complex

$$
0 \longrightarrow C_{H}^{0}(\Omega, E) \stackrel{d^{0}}{\longrightarrow} C_{H}^{1}(\Omega, E) \stackrel{d^{1}}{\longrightarrow} \ldots
$$

where $C_{H}^{n}(\Omega, E)=\operatorname{Hom}_{\Omega}\left(B \times \mathbf{R}, F^{n}(\Omega, E)\right)$ and $d_{*}^{n}$ is abbreviated to $d^{n}$.

We regard elements of $C_{H}^{n}(\Omega, E)$ as invariant sections of $F^{n}(\Omega, E)$, that is, as maps

$$
\varphi: \bigcup_{x \in B} \Omega^{n+1} \rightarrow E
$$

which commute with the projections and satisfy the homogeneity condition

$$
\varphi_{x}\left(\eta_{0} \xi, \ldots, \eta_{n} \xi\right)=\xi^{-1} \cdot \varphi_{y}\left(\eta_{0}, \ldots, \eta_{n}\right)
$$

for $\eta_{j} \in \Omega_{y}, \xi \in \Omega_{x}^{y}$ and $x, y \in B$. Such maps are automatically continuous (given that each $\varphi_{x}: \Omega_{x}^{n+1} \rightarrow E_{x}$ is) since invariant sections of a CVB are automatically continuous.

In practice it is easier to work with nonhomogenous cochains, which are defined in the usual way. (We omit most proofs of continuity considerations from this point the verifications following an established pattern.)

For $n \geqslant 0$,

$$
*^{n} \Omega=\left\{\left(\xi_{1}, \ldots, \xi_{n}\right) \in \Omega^{n} \mid a \xi_{j}=b \xi_{j+1} \text { for } 1 \leqslant j<n\right\}
$$

denotes the set of composable $n$-tuples from $\Omega$ with the subspace topology. It forms a fibre bundle over $B$ with respect to the projection $\left(\xi_{0}, \ldots, \xi_{n}\right) \rightarrow a \xi_{n}$. Given a continuous $\Omega$-module $E$ one can form a CVB whose fibre over $x \in B$ consists of all continuous maps from $\left.*^{n} \Omega\right|_{x}$ to $E_{x}$, and the continuous sections of this CVB are the nonhomogeneous cochains:

Definition 5.6. $C_{N H}^{n}(\Omega, E)$, the LCS of nonhomogeneous n-cochains, is the set of all maps $f: *^{n} \Omega \rightarrow E$ such that $p\left(f\left(\xi_{1}, \ldots, \xi_{n}\right)\right)=a \xi_{n}$, continuous as defined above, and with the compact-open topology as a space of sections. 
Define $\delta^{n}: C_{N H}^{n}(\Omega, E) \rightarrow C_{N H}^{n+1}(\Omega, E)$ by

$$
\begin{aligned}
\left(\delta^{n} f\right)\left(\xi_{1}, \ldots, \xi_{n+1}\right) & \\
=f\left(\xi_{2}, \ldots, \xi_{n+1}\right) & +\sum_{j+1}^{n}(-1)^{i} f\left(\xi_{1}, \ldots, \xi_{,} \xi_{j+1}, \ldots, \xi_{n+1}\right) \\
& +(-1)^{n+1} \xi_{n+1}^{-1} . f\left(\xi_{1}, \ldots, \xi_{n}\right)
\end{aligned}
$$

for $\left(\xi_{1}, \ldots, \xi_{n+1}\right) \in *^{n+1} \Omega$. As in the group case, we have

Proposition 5.8. There are linear homeomorphisms $T^{n}: C_{H}^{n}(\Omega, E) \rightarrow C_{N H}^{n}(\Omega, E)$ such that $\delta^{n} \circ T^{n}=T^{n+1} \circ d^{n}(n \geqslant 0)$, given by

$$
\begin{gathered}
T^{n}(p)\left(\xi_{1}, \ldots, \xi_{n}\right)=\varphi\left(\xi_{1}, \ldots \xi_{n}, \xi_{2}, \ldots, \xi_{n}, \ldots, \xi_{n}, \widetilde{a} \xi_{n}\right), \\
\left(T^{n}\right)^{-1}(f)\left(\xi_{0}, \ldots, \xi_{n}\right)=\xi_{n}^{-1}, f\left(\xi_{0} \cdot \xi_{1}^{-1}, \xi_{1} \cdot \xi_{2}^{-1}, \ldots, \xi_{n} \cdot \xi_{n+1}^{-1}\right) .
\end{gathered}
$$

For most purposes we will regard $H^{n}(\Omega, E)$ as $\operatorname{ker} \delta^{n} / \mathrm{im} \delta^{n-1}$ and ne denote $C_{N H}^{n}(\Omega, E)$ simply by $C^{n}(\Omega, E)$.

When $n=0$, we identify $*^{n} \Omega$ with $B$, so that $C^{0}(\Omega, E)$ is identified with $\Gamma E$. Accordingly, (5.7) becomes

$$
\delta^{\wedge}(\mu)(\xi)=\mu(a \xi)-\xi^{-1} \cdot \mu(b \xi), \quad \text { for } \mu \in \Gamma E=C^{0}(\Omega, E), \quad \xi \in \Omega,
$$

and (5.8) becomes

$$
T^{0}(\tilde{\xi})(x)=\varphi(\tilde{x}), \quad x \in B, \quad \tilde{p} \in C_{l i}^{0}(\Omega, E),
$$

and

$$
\left(T^{0}\right)^{-1}(\mu)(\xi)=\xi^{-1} \cdot \mu(b \xi), \quad \xi \in \Omega, \quad \mu \in C^{0}(\Omega, E) .
$$

\section{Extensions of topological groupoids}

In (4.1) and (4.5) $H^{\circ}(\Omega, E)$ was described as the space of invariant sections of $E$, and shown to be naturally isomorphic to any $H^{0}\left(\Omega_{w}^{w}, E_{w}\right)$. In Section 7 we will prove that $H^{n}(\Omega, E) \cong H^{n}\left(\Omega_{w}^{w}, E_{w}\right)$ for all $n \geqslant 0$, but first we study the extensions classified by $H^{2}(\Omega, E)$ and use them to interpret $H^{1}(\Omega, E)$.

As always, we work over a base $B$ which is a paracompact connected $C^{0}$ manifold. 
DefintTion 6.1. If $\Omega$ is a topological groupoid on $B$ and $(E, p, B)$ is a CVB then a general extension of $\Omega$ by $E$ is a topological groupoid $\Phi$ on $B$ and an exact sequence

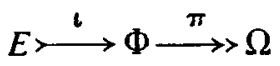

in which $\iota$ is a relatively open continuous morphism and $\pi$ is a topological submersion and a continuous morphism.

REMARKS. (i) A topological submersion is a continuous onto map which admits local right-inverses at every point of its range (Hardy (1971)).

(ii) That $\iota$ is a continuous morphism means that $\iota$ is continuous, maps each $E_{x}$ into $\Phi_{x}^{x}$ and $\iota(u+v)=\imath(u) \iota(v)$ for all $u, v \in E_{x}, x \in B$.

(iii) That (6.1) is exact means that $\pi$ is onto, $\iota$ is $1: 1$ and, for $\zeta \in \Phi, \pi(\zeta) \in B \subset \Omega$ if and only if $\zeta=\imath(u)$ for some $u \in E$. In particular, each vertex sequence $E_{x} \gg \Phi_{x}^{x} \rightarrow \Omega_{x}^{x}$ is exact.

This is the most general viable concept of topological groupoid extension; it satisfies the minimal requirements of inducing a continuous action of $\Omega$ on $E$, and permitting a $1: 1$ correspondence between left and right transversals (when they exist).

Clearly each $E_{x} \hookrightarrow \Phi_{x}^{x} \rightarrow \Omega_{x}^{x}$ is an extension of the topological group $\Omega_{x}^{x}$ by $E_{x}$; it can be easily verified that each inner isomorphism $\Phi_{x}^{x} \rightarrow \Phi_{y}^{y}, \xi \rightarrow \zeta . \xi . \zeta^{-1}$ for $\zeta \in \Phi_{x}^{y}$ induces an isomorphism of $E_{x} \gg \Phi_{x}^{x} \rightarrow \Omega_{x}^{x}$ with $E_{y} \longrightarrow \Phi_{y}^{y} \rightarrow \Omega_{y}^{y}$.

Proposition 6.2. The general extension (6.1) induces a continuous, but not necessarily linear, action of $\Omega$ on $E$ by

$$
\iota(\pi(\zeta) \cdot u)=\zeta . \iota(u) . \zeta^{-1} \text { for } \zeta \in \Phi_{x}^{\nu}, \quad u \in E_{x}, \quad x, y \in B .
$$

(That is, (6.2) satisfies all the conditions of (3.1) except that $E_{x} \rightarrow E_{y}$ need not be linear.)

Proof. If $G \Phi$ denotes the bundle of vertex groups of $\Phi$, that is, $G \Phi=\bigcup_{x \in B} \Phi_{x}^{x}$ with the subspace topology, then $\Phi * G \Phi \rightarrow G \Phi,(\zeta, \lambda) \rightarrow \zeta . \lambda . \zeta^{-1}$ is continuous, and induces $\Phi * E \rightarrow \imath(E) \subset G \Phi$. Since $\iota$ is relatively open $\Phi * E \rightarrow E$ is continuous. If now $\sigma: U \subset \Omega \rightarrow \Phi$ is a local section of $\pi$ then $\sigma * \mathrm{id}_{E_{\alpha(U)}}: U * E_{a(U)} \rightarrow \Phi * E$ is a local section of $\pi * \mathrm{id}_{E}$. Hence $\pi * \mathrm{id}_{E}$ has local sections and is therefore an identification. Since $\Omega * E \rightarrow E$ composed with $\pi * \mathrm{id}_{E}$ is the continuous $\Phi * E \rightarrow E$ it follows that (6.2) is continuous. 
Definition 6.3. A transversal (or right-transversal) of the general extension (6.1) is a continuous map $n: \Omega \rightarrow \Phi$ such that $\pi \circ n=\mathrm{id}_{\Omega}$. A left-transversal is a continuous $m: \Phi \rightarrow E$ such that $m \circ \iota=\mathrm{id}_{E}$.

Since $\iota$ and $\pi$ are initial and final maps respectively, the following result is proved by an algebraic manipulation.

PROPOSITION 6.4. If $n$ is a right-transversal of (6.1) then $m: \Phi \rightarrow E$ defined by $\iota(m(\zeta))=n(\pi(\zeta))^{-1} . \zeta, \zeta \in \Phi$, is a left transversal. Conversely, if $m$ is a lefttransversal, then $n: \Omega \rightarrow \Phi$ defined by $n(\pi(\zeta))=\zeta .\left(\llcorner\circ m(\zeta))^{-1}, \zeta \in \Phi\right.$, is a well-defined right-transversal.

The crucial property of an extension is, of course, whether it admits transversals. Thus we make the

DefinItIon 6.5. The general extension (6.1) is rigid if it admits a global transversal $n: \Omega \rightarrow \Phi$. It is locally rigid if there is an open cover $\left\{U_{\alpha}\right\}$ of $B$ and local transversals $n_{\alpha}: \Omega_{U_{\alpha}^{\alpha}}^{U} \rightarrow \Phi_{U_{\alpha}^{\alpha}}^{U_{\alpha}}$ over each $U_{\alpha}$.

Observe that in a locally rigid extension $E \nrightarrow \Phi \rightarrow \Omega$ each vertex extension $E_{x} \longleftrightarrow \Phi_{x}^{x} \rightarrow \Omega_{x}^{x}$ has a global transversal and can therefore be described by the Hochschild-Mostow cohomology (Theorem 3). In studying the question of global extensions for groupoids it is natural to assume that on the vertex group level the extension is well behaved and so the following proposition demonstrates that local rigidity is the appropriate concept in the context of locally trivial groupoids.

Proposition 6.6. (i) If, in the general extension (6.1) both $\Omega$ and $\Phi$ are locally trivial and the vertex group extensions admit transversals $\Omega_{x}^{x} \rightarrow \Phi_{x}^{x}$ then the extension is locally rigid.

(ii) A general extension of a locally trivial groupoid is locally trivial.

Proof. (i) If $n: \Omega_{w}^{w} \rightarrow \Phi_{w}^{w}$ is a vertex group transversal over $w \in B$ and $\left\{\tau_{\alpha}: U_{\alpha} \rightarrow \Phi_{w}\right\}$ is a family of decomposing sections for $\Phi$, then $n_{\alpha}: \Omega_{U_{\alpha}}^{U_{\alpha}} \rightarrow \Phi_{U_{\alpha}^{\alpha}}^{U_{\alpha}}$ defined by $n_{\alpha}(\xi)=\tau_{\alpha}(b \xi) \cdot n\left(\sigma_{\alpha}(b \xi)^{-1} \cdot \xi \cdot \sigma_{\alpha}(a \xi)\right) \cdot \tau_{\alpha}(a \xi)^{-1}$, where $\sigma_{\alpha}=\pi \circ \tau_{\alpha}$, is a local transversal over $U_{\alpha}$.

(ii) If $E \stackrel{\iota}{\longrightarrow} \Phi \stackrel{\pi}{\longrightarrow}>\Omega$ is a general extension of the locally trivial topological groupoid $\Omega$, then $[b, a]_{\Phi}=[b, a]_{\Omega} \circ \pi$ and since both $[b, a]_{\Omega}$ and $\pi$ are topological submersions, it follows that $[b, a]_{\Phi}$ is also.

Proposition 6.7. In any locally rigid extension $E \succ \Phi \rightarrow \Omega$ of the locally trivial $\Omega$, there are continuous maps $n_{x}: \Omega_{x} \rightarrow \Phi_{x}$ with $\pi \circ n_{x}=\mathrm{id}_{\Omega_{x}}$ for each $x \in B$. 
Proof. (Observe that local rigidity makes $\Phi_{x}$ a principal fibre bundle orer $\Omega_{x}$ with vector structure group $E_{x}$ and as in the finite dimensional case, it is therefore trivial.) There exists an open cover $\left\{U_{\alpha}\right\}$ of $B$ with decomposing sections $\tau_{\alpha}: U_{\alpha} \rightarrow \Phi_{\omega}$ and local transversals $n_{\alpha}: \Omega_{U_{\alpha}^{\alpha}}^{U_{\alpha}} \rightarrow \Phi_{U_{\alpha}^{\alpha}}^{U_{\alpha}}$. Then define $n_{\alpha, w}: \Omega_{w_{\alpha} \rightarrow \Phi_{w}^{U}}^{U}$ by

$$
n_{\alpha, w}(\xi)=n_{\alpha}\left(\xi \cdot\left(\pi \circ \tau_{\omega}\right)(b \xi)^{-1}\right) \cdot \tau_{\alpha}(b \xi)
$$

and $g_{\alpha \beta}: \Omega_{w}^{U_{\alpha \beta} \rightarrow E_{w}}$ by

$$
\iota\left(g_{\alpha \beta}(\xi)\right)=n_{\alpha, w}(\xi)^{-1} \cdot n_{\beta, w}(\xi) .
$$

Since $B$ is paracompact there is a partition of unity $\left\{j_{\alpha}\right\}$ subordinate to $\left\{U_{\alpha}\right\}$ and $n_{w}: \Omega_{w} \rightarrow \Phi_{w}$ defined by

$$
n_{w}(\xi)=n_{\alpha, w}(\xi) \cdot\left(\sum_{\beta} j_{\beta}(b \xi) g_{\alpha \beta}(\xi)\right)
$$

now has the desired properties.

Since $n_{w}$ has no multiplicative properties, it is not possible to extend it to $\Omega \rightarrow \Phi$. However, we will deduce in Section 7 that in fact all locally rigid extensions of locally trivial groupoids are rigid.

From now to the end of Section 6 , let $\Omega$ be a locally trivial, locally compact topological groupoid on $B$ and let $(E, p, B)$ be a continuous $\Omega$-module.

Definition 6.8. A general extension $E \stackrel{\iota}{\longrightarrow} \Phi \stackrel{\pi}{\longrightarrow} \Omega$ is an operator extension of $\Omega$ by the continuous $\Omega$-module $E$ if the action of $\Omega$ on $E$ induced by the extension coincides with the module action.

Two operator extensions $E \stackrel{\iota_{j}}{\longrightarrow} \Phi_{j} \stackrel{\pi_{j}}{\longrightarrow} \Omega_{j}, j=1,2$, are equivalent if there exists a continuous morphism (necessarily an isomorphism) $\varepsilon: \Phi_{1} \rightarrow \Phi_{2}$ such that $\varepsilon \circ \iota_{1}=\iota_{2}$ and $\pi_{2} \circ \varepsilon=\pi_{1}$.

The set of equivalence classes of operator extensions is denoted by Opext $(\Omega, E)$; clearly equivalence preserves transversals so we have the subsets LocROpext $(\Omega, E)$ of locally rigid operator extensions and $\operatorname{ROpext}(\Omega, E)$ of rigid operator extensions.

One can show, in a straightforward fashion, that $\operatorname{Opext}(\Omega, E)$ forms an abelian group under a Baer sum, and that $\operatorname{LocROpext}(\Omega, E)$ and $\operatorname{ROpext}(\Omega, E)$ are subgroups. If $\Phi$ and $\Psi$ are groupoids over $B$ then their "Whitney product" $\Phi \Delta \Psi$ is the groupoid over $B$ with $(\Phi \Delta \Psi)_{x}^{y}=\Phi_{x}^{y} \times \Psi_{x}^{\nu}$ for $x, y \in B$ and componentwise multiplication. When $\Phi$ and $\Psi$ are topological groupoids, $\Phi \Delta \Psi$ is also, with the relative product topology. Push-outs and pull-backs do not exist in general in the locally trivial context, so it is necessary to use the older definition of the Baer sum: given $E \stackrel{\iota_{1}}{\longrightarrow} \Phi_{1} \stackrel{\pi_{1}}{\longrightarrow} \Omega \Omega$ and $E \stackrel{\iota_{2}}{\longrightarrow} \Phi_{2} \stackrel{\pi_{2}}{\longrightarrow}, \Omega$, one considers the subgroupoid 
$\left\{\left(\zeta_{1}, \zeta_{2}\right) \mid \pi_{1} \zeta_{1}=\pi_{2} \zeta_{2}\right\}$ of $\Phi_{1} \Delta \Phi_{2}$ and quotients it over $\left\{\left(\iota_{1}(u), \iota_{2}(-u) \mid u \in E\right\}\right.$. When the given extensions are locally rigid, one then shows easily that this quotient groupoid is locally trivial, and so (6.6(i)) shows that the sum of the extensions is locally rigid.

There is now a morphism

$$
\mathscr{V}: \operatorname{LocROpext}(\Omega, E) \rightarrow \operatorname{ROpext}\left(\Omega_{w}^{w}, E_{w}\right)
$$

which sends $E \succ \Phi \rightarrow \Omega$ to the vertex group extension $E_{w} \longrightarrow \Phi_{w}^{w} \rightarrow \Omega_{w}^{w}$.

THEOREM 2. Let $\Omega$ be a locally trivial, locally compact topological groupoid on $B$, and let $(E, p, B)$ be a continuous $\Omega$-module.

(i) Let $f$ be a nonhomogeneous 2-cocyle, thus $f: *^{2} \Omega \rightarrow E$ continuously, $f\left(\xi_{1}, \xi_{2}\right) \in E_{a \xi_{2}}$ and $f\left(\xi_{2}, \xi_{3}\right)+f\left(\xi_{1}, \xi_{2} \cdot \xi_{3}\right)=f\left(\xi_{1} \cdot \xi_{2}, \xi_{3}\right)+\xi_{3}^{-1} \cdot f\left(\xi_{1}, \xi_{2}\right)$ for all

$$
\left(\xi_{1}, \xi_{2}, \xi_{3}\right) \in *^{3} \Omega \text {. }
$$

Then if $\Phi_{f}$ is the pull-back $\Omega * E=\{(\xi, u) \mid a \xi=p u\}$ and we define source and target maps $\vec{a}(\xi, u)=p u, \vec{b}(\xi, u)=b \xi$ and a composition

$$
\left(\xi_{1}, u_{1}\right) \cdot\left(\xi_{2}, u_{2}\right)=\left(\xi_{1} \cdot \xi_{2}, u_{2}+\xi_{2}^{-1} \cdot u_{1}+f\left(\xi_{1}, \xi_{2}\right)\right)
$$

then $\Phi_{f}$ is a locally trivial topological groupoid on $B$. The identities are

$$
\tilde{x}=(\tilde{x},-f(\tilde{x}, \tilde{x})) \text { and }(\xi, u)^{-1}=\left(\xi^{-1},-\xi \cdot u-f\left(\xi, \xi^{-1}\right)-f(\widetilde{b \xi}, \widetilde{b \xi})\right) \text {. }
$$

With $\pi_{f}: \Phi_{f} \rightarrow \Omega$ by $\pi_{f}(\xi, u)=\xi$ and $\iota_{f}: E \rightarrow \Phi_{f}$ by $\iota_{f}(u)=(\widetilde{p u}, u-f(\widetilde{p u}, \widetilde{p u}))$, $E \stackrel{\pi_{f}}{\longrightarrow} \Phi_{f} \stackrel{l_{f}}{\longrightarrow} \Omega$ becomes an operator extension of $\Omega$ by $E$, and $n_{f}(\xi)=\left(\xi, 0_{a \xi}\right)$ defines a global transversal $n_{f}: \Omega \rightarrow \Phi_{f}$. Note that $\Phi_{f}$ is locally compact if and only if $E$ is of finite rank.

(ii) Conversely, if $E \stackrel{\iota}{\longrightarrow} \Phi \stackrel{\pi}{\longrightarrow}>\Omega$ is a rigid operator extension with transversal $n: \Omega \rightarrow \Phi$ and we define $f: *^{2} \Omega \rightarrow E$ by $،\left(f\left(\xi_{1}, \xi_{2}\right)\right)=n\left(\xi_{1} \xi_{2}\right)^{-1} n\left(\xi_{1}\right) n\left(\xi_{2}\right)$ then $f$ is a nonhomogeneous 2-cocycle, and the map $\mathscr{E}_{n}: \Phi_{f} \rightarrow \Phi,(\xi, u) \rightarrow n(\xi) . \iota(u)$ is an equivalence of $E \stackrel{\iota}{\longrightarrow} \Phi \stackrel{\pi}{\longrightarrow}>\Omega$ with $E \stackrel{\iota_{f}}{\longrightarrow} \Phi_{f} \stackrel{\pi_{f}}{\longrightarrow}>\Omega$ preserving transversals, $\mathscr{E}_{n} \circ n_{f}=n$. On the other hand, the cocycle defined by $n_{f}$ is $f$ itself.

For $f$ and $f^{\prime}$ both 2-cocycles, there is a natural equivalence from $\Phi_{f+f^{\prime}}$ to the Baer sum of $\Phi_{f}$ and $\Phi_{f}$.

(iii) Let $g$ be a nonhomogeneous 1-cochain, thus $g: \Omega \rightarrow E$ is continuous, $p \circ g=a$, and

$$
\delta g\left(\xi_{1}, \xi_{2}\right)=g\left(\xi_{2}\right)-g\left(\xi_{1} \cdot \xi_{2}\right)+\xi_{2}^{-1} \cdot g\left(\xi_{1}\right)
$$


for $\left(\xi_{1}, \xi_{2}\right) \in *^{2} \Omega$. Given any 2-cocycle $f$, the map $\varepsilon_{g}: \Phi_{f} \rightarrow \Phi_{f-\delta g},(\xi, u)+(\xi, u+g(\xi))$ is an equivalence of $E_{\succ} \rightarrow \Phi_{f \rightarrow} \rightarrow \Omega$ with $E \succ \Phi_{f-\delta g} \rightarrow \Omega$.

(iv) Conversely, if $E \stackrel{\iota^{\prime}}{\longrightarrow} \Phi^{\prime} \stackrel{\pi^{\prime}}{\longrightarrow} \Omega$ and $E \stackrel{\iota}{\longrightarrow} \Phi \stackrel{\pi}{\longrightarrow} \Omega$ are rigid operator extensions with transversals $n^{\prime}$ and $n$ defining cocycles $f^{\prime}$ and $f$ and $\varphi: \Phi \rightarrow \Phi^{\prime}$ is an equivalence, then there is a (unique) 1-cochain $g$ such that $\varphi=\mathscr{E}_{n}^{\prime} \circ \varepsilon_{g} \circ \mathscr{E}_{n}^{-1}$, namely $g=m^{\prime} \circ \varphi \circ n$, and $\delta g=f-f^{\prime}$.

Indeed each 1-cochain $g$ induces a permutation of the transversals in any given extension. If $E \stackrel{\iota}{\longrightarrow} \Phi \stackrel{\pi}{\longrightarrow}>\Omega$ is a rigid extension and $n$ is a transversal for it, then $n^{g}$ defined by $n^{g}(\xi)=n(\xi) \iota(g(\xi)), \xi \in \Omega$, is another and defines the cocycle $f+\delta g$, where $f$ is the cocycle defined by $n$. The different equivalences are related by $\mathscr{E}_{n \sigma}=\mathscr{E}_{n} \circ \varepsilon_{g}$.

Proof. This is a straightforward verification; the algebraic computations follow closely the corresponding results for groups (see, for example, MacLane (1963)) and the topological considerations are of a type considered already.

COROLLARY 6.10. The assignment $f \rightarrow \Phi_{f}$ of the theorem induces an isomorphism $H^{2}(\Omega, E) \rightarrow \operatorname{ROpext}(\Omega, E)$.

Needless to say, the topology of $H^{2}(\Omega, E)$ can be transferred to ROpext $(\Omega, E)$.

Proposition 6.11. If $E \stackrel{\iota}{\longrightarrow} \Phi \stackrel{\pi}{\longrightarrow}>\Omega$ is a rigid extension of the locally trivial, locally compact topological groupoid $\Omega$ then an open cover of $B$ will decompose $\Omega$ if and only if it decomposes $\Phi$. If $\left\{U_{\alpha}\right\}$ decomposes $\Omega$ through $\left\{\sigma_{\alpha}: U_{\alpha} \rightarrow \Omega_{w}\right\}$ with transition functions $\left\{s_{\alpha \beta}\right\}$ and $n: \Omega \rightarrow \Phi$ is a transversal defining the cocycle $f$, then

$$
t_{\alpha \beta}(x)=n\left(s_{\alpha \beta}(x)\right) . \iota\left(f\left(\sigma_{\alpha}(x), s_{\alpha \beta}(x)\right)\right)^{-1}, \quad x \in U_{\alpha \beta},
$$

are transition functions for $\Phi$.

PRoOF. By (1.5) we know that if $\left\{U_{\alpha}\right\}$ decomposes $\Phi$ through $\left\{\tau_{\alpha}: U_{\alpha} \rightarrow \Phi_{w}\right\}$ then $\left\{\pi \circ \tau_{\alpha}: U_{\alpha} \rightarrow \Omega_{w}\right\}$ will decompose $\Omega$. We need only note that $\varphi$ need not be a morphism in (1.5) but need only preserve the source and target maps, so the same remark applies to $n: \Omega \rightarrow \Phi$. The formula is a straightforward calculation of $t_{\alpha \beta}(x)=n\left(\sigma_{\alpha}(x)\right)^{-1} \cdot n\left(\sigma_{\beta}(x)\right)$ using Theorem 2(ii).

Lastly, $H^{1}(\Omega, E)$ can be interpreted as automorphisms of the zero extension modulo those inner automorphisms which are induced by sections of $E$, in the usual way. 


\section{Relationship with the vertex group cohomology}

Throughout Section $7, \Omega$ is a locally trivial, locally compact topological groupoid on base $B$, unless explicitly described otherwise.

Choosing a $w \in B$ there is a natural restriction functor $\mathscr{R}: \Omega$-Mod $\rightarrow G$-Mod, where $G=\Omega_{w}^{w}$, which takes a continuous $\Omega$-module $E$ to $E_{w}$ and a continuous $\Omega$-module morphism $\varphi: E^{1} \rightarrow E^{2}$ to $\varphi_{w}: E_{w}^{1} \rightarrow E_{w}^{2}$.

The mixing process also allows us to define a functor $\mathscr{M}: G$-Mod $\rightarrow \Omega-\operatorname{Mod}$ by assigning to $A \in G$-Mod the fibre bundle associated to $\Omega_{w}(B, G)$ with respect to the module action of $G$ on $A$. The construction is given by Seda (1975) but we review the notation.

The set $\mathscr{K}(A)$ is the set of equivalence classes $(\overline{\xi, u})$ with $\xi \in \Omega_{w}$ and $u \in A$, where $(\xi . g, u) \sim(\xi, g u)$ for $g \in G$ is the equivalence; $\mathscr{M}(A)$ has the identification topology from the product space $\Omega_{w} \times A$. The bundle projection is $(\overline{\xi, u}) \rightarrow b \xi$ and the operations in $E_{x}$ are $\left.\lambda . \overline{(\xi, u}\right)=(\overline{\xi, \lambda u})$ and $\left(\overline{\xi_{1}, u_{1}}\right)+\left(\overline{\xi_{2}, u_{2}}\right)=\left(\overline{\xi_{1}, u_{1}+\xi_{1}^{-1} \cdot \xi_{2} u_{2}}\right)$ (here $\lambda \in \mathbf{R}$ ). Then $\mathscr{M}(A) \rightarrow B$ is a CVB and a continuous $\Omega$-module under the action $\zeta \cdot(\overline{\xi, u})=(\overline{\zeta \xi, u})$.

If $\varphi: A_{1} \rightarrow A_{2}$ is a continuous $G$-module morphism then $(\overline{\xi, u}) \rightarrow(\overline{\xi, \varphi(u)})$ is well defined and is in fact a continuous $\Omega$-module morphism $\mathscr{M}(\varphi): \mathscr{M}\left(A_{1}\right) \rightarrow \mathscr{M}\left(A_{2}\right)$.

\section{Proposition 7.1. $\mathscr{M}$ and $\mathscr{R}$ are adjoint.}

Proof. The core of this is proved by Seda (1975). We only give an outline. For $A \in G$-Mod define $\varepsilon_{A}: A \rightarrow \mathscr{R} \circ \mathscr{K}(A)$ by $u \rightarrow(\overline{\tilde{w}, u})$ and for $E \in \Omega$-Mod define $\theta_{E}: \mathscr{M} \circ \mathscr{R}(E) \rightarrow E$ by $(\overline{\xi, u}) \rightarrow \xi . u$. Then Seda proves that $\varepsilon$ and $\theta$ are natural equivalences id $_{G \text {-Mod }} \rightarrow \mathscr{R} \circ \mathscr{M}$ and $\mathscr{M} \circ \mathscr{R} \rightarrow$ id $_{\Omega \text {-Mod }}$ respectively, and one easily verifies that $\theta_{\mathscr{K}(A)} \circ \mathscr{M}\left(\varepsilon_{A}\right)=\mathrm{id} \mathcal{H}_{(A)}$ and $\mathscr{R}\left(\theta_{E}\right) \circ \varepsilon_{\mathscr{R}(E)}=\mathrm{id}_{\mathscr{R}(E)}$ for all $A \in G$-Mod and $E \in \Omega$-Mod.

LemMA 7.2. Both $\mathscr{R}: \Omega$-Mod $\rightarrow G$-Mod and $\mathscr{M}: G$-Mod $\rightarrow \Omega$-Mod are exact and preserve (continuous) injectives.

Proof. That $\mathscr{R}$ is exact is trivial. Let

$$
A_{1} \stackrel{\iota}{\stackrel{\iota}{\rightleftarrows}} A_{2} \underset{\rho}{\stackrel{\pi}{\rightleftarrows}} A_{3}
$$

be exact in $G$-Mod, then applying $\mathscr{N}$ we get

$$
\mathscr{M}\left(A_{1}\right) \stackrel{\mathscr{K}(\iota)}{\longrightarrow} \mathscr{M}\left(A_{2}\right) \stackrel{\mathscr{M}(\pi)}{\longrightarrow} \mathscr{M}\left(A_{3}\right)
$$


and it has to be shown that $\lambda$ and $\rho$ can be globalized. If $\left\{U_{\alpha}\right\}$ decomposes $\Omega$ through $\left\{\sigma_{\alpha}: U_{\alpha} \rightarrow \Omega_{w}\right\}$ and $\left\{j_{\alpha}\right\}$ is a partition of unity subordinate to $\left\{U_{\alpha}\right\}$ then

$$
\left.\bar{\lambda}\left(\overline{\xi, u_{2}}\right)=\sum_{\alpha} j_{\alpha}(b \xi) \cdot\left(\overline{\sigma_{\alpha}(b \xi), \lambda\left(\sigma_{\alpha}(b \xi)^{-1} \cdot \xi \cdot u_{2}\right.}\right)\right)
$$

and

$$
\left.\left.\bar{\rho}\left(\overline{\xi, u_{3}}\right)=\sum_{\alpha} j_{\alpha}(b \xi) \cdot \overline{\left(\sigma_{\alpha}(b \xi), \rho\left(\sigma_{\alpha}(b \xi)^{-1} \cdot \xi \cdot u_{3}\right.\right.}\right)\right)
$$

are CVB morphisms and clearly $\mathscr{M}(\pi) \circ \bar{\rho}$ and $\bar{\lambda} \circ \mathscr{M}(\iota)$ are identities. One also sees that

$$
\begin{aligned}
(\bar{\rho} \circ \mathscr{K}(\pi)+\mathscr{M}(\iota) \circ \bar{\lambda})\left(\overline{\xi, u_{2}}\right) \\
\left.=\sum_{\alpha} j_{\alpha}(b \xi) \cdot\left(\overline{\sigma_{\alpha}(b \xi),\left(\sigma_{\alpha}(b \xi)^{-1} \cdot \xi \cdot \pi\left(u_{2}\right)\right)+\iota\left(\lambda\left(\sigma_{\alpha}(b \xi)^{-1} \cdot \xi \cdot u_{2}\right)\right.}\right)\right) \\
\left.=\sum_{\alpha} j_{\alpha}(b \xi) \cdot\left(\overline{\sigma_{\alpha}(b \xi),(\rho \circ \pi+\imath \circ \lambda)\left(\sigma_{\alpha}(b \xi)^{-1} \cdot \xi \cdot u_{2}\right.}\right)\right) \\
=\left(\overline{\xi, u_{2}}\right)
\end{aligned}
$$

from which $\bar{\lambda}_{\circ} \tilde{\rho}=0$ follows. Hence $\mathscr{M}$ is exact.

Now let $E$ be a continuously injective $\Omega$-module, and let $\varphi: A_{1} \rightarrow E_{w}$ be a continuous $G$-module morphism, and

$$
\psi: A_{1} \rightleftarrows A_{2}
$$

a $G$-monomorphism. By the above, $\mathscr{M}(\psi)$ is an $\Omega$-monomorphism, so $\theta_{E} \circ \mathscr{M}(\varphi)$ has a lifting to a continuous $\Omega$-module morphism $\mu: \mathscr{K}\left(A_{2}\right) \rightarrow E$ and then $\mu_{w} \circ \varepsilon_{A_{2}}$ lifts $\varphi$ showing that $E_{w}$ is continuously injective.

Similarly $\mathscr{R}$ preserves injectives.

THEOREM 3. For any continuous $\Omega$-module $E$, where $\Omega$ is a locally trivial, locally compact topological groupoid on $B$, and $w \in B$, there are natural linear homeomorphisms

$$
H^{n}(\Omega, E) \rightarrow H^{n}\left(\Omega_{w}^{w}, E_{w}\right) \text { for each } n \geqslant 0 .
$$

Proof. Consider the $H^{n}\left(\Omega_{w}^{w},-\right) \circ \mathscr{R}$ as functors from $\Omega$-Mod to the category of LCS's. Using the exactness of $\mathscr{R}$, one easily sees that they form a connected sequence of functors, and since $\mathscr{R}$ preserves injectives, they vanish on the continuously injective $\Omega$-modules for $n>0(4,4)$. In Section 4 we showed that the same was true of the $H^{n}(\Omega,-)$ and so the natural linear homeomorphism

$$
H^{0}(\Omega,-) \rightarrow H^{0}\left(\Omega_{w}^{w},-\right) \circ \mathscr{R}
$$


of (4.5) extends to unique linear homeomorphisms in all dimensions, by the second uniqueness theorem of Lang (1966). This completes the proof.

CoRollary 7.3. $\mathscr{V}:$ LocROpext $(\Omega, E) \rightarrow \operatorname{ROpext}\left(\Omega_{w}^{w}, E_{w}\right)$ is onto.

Proof. By Theorem 2, applied with $B=\{w\}$, an element of ROpext $\left(\Omega_{w}^{w}, E_{w}\right)$ defines an element of $H^{2}\left(\Omega_{w}^{w}, E_{w}\right)$ and thus, by Theorem 3, an element of $H^{2}(\Omega, E)$. Theorem 2 again now gives an element of $\operatorname{ROpext}(\Omega, E) \subset \operatorname{LocROpext}(\Omega, E)$ which is easily seen to restrict over $w$ to the original extension.

In fact there is a commutative square

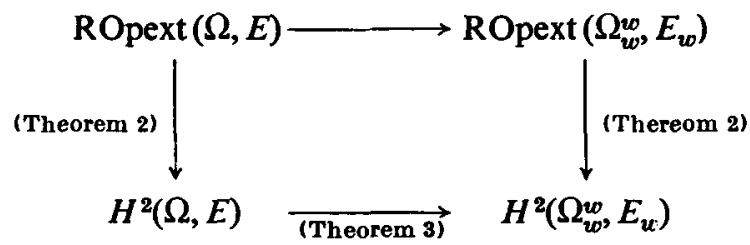

in which the two vertical maps and the lower horizontal one are isomorphisms by the theorems indicated, and so the top map, which is the restriction of $\mathscr{V}$, is also an isomorphism. We thus have

COROLLARY 7.4. A rigid operator extension of $\Omega$ by $E$ is completely determined by its restriction to any vertex.

Clearly the kernel of $\mathscr{V}$ consists of those locally rigid operator extensions which are semi-direct on the vertex group level. But in fact the method of (6.7) adapts to show that such extensions are globally semi-direct.

Proposition 7.5. Let $E$ be a continuous $\Omega$-module, where $\Omega$ is a locally trivial, locally compact topological groupoid on $B$, and let $E \stackrel{\iota}{\longrightarrow} \Phi \stackrel{\pi}{\longrightarrow} \Omega$ be a locally rigid operator extension with a morphism of topological groups $n_{w}: \Omega_{w}^{w} \rightarrow \Phi_{w}^{w}$ such that $\pi \circ n_{w}=\mathrm{id}_{\Omega^{\text {r. }}}$. Then $E \stackrel{\iota}{\longrightarrow} \Phi \stackrel{\pi}{\longrightarrow} \Omega$ is globally semi-direct.

Proof. $\Phi$ is locally trivial by (6.6(ii)); let $\left\{\tau_{\alpha}: U_{\alpha} \rightarrow \Phi_{w}\right\}$ be a family of decomposing sections for $\Phi$. Define $n_{\alpha}: \Omega_{v}^{U_{\alpha}} \rightarrow \Phi_{v}^{U_{\alpha}}$ by

$$
n_{\alpha}(\xi)=\tau_{\alpha}(b \xi) \cdot n_{v o}\left(\pi\left(\tau_{\alpha}(b \xi)\right)^{-1} \cdot \xi\right)
$$

and note that $n_{\alpha}(\xi . g)=n_{\alpha}(\xi) \cdot n_{w}(g)$ for $g \in \Omega_{w}^{w}$ and $\xi \in \Omega_{w}^{U_{\alpha}}$. Now define $f_{\alpha \beta}: \Omega_{w}^{U_{\alpha \beta} \rightarrow E_{w}}$ by $\iota\left(f_{\alpha \beta}(\xi)\right)=n_{\alpha}(\xi)^{-1} \cdot n_{\beta}(\xi)$ and note that $f_{\alpha \beta}(\xi . g)=g^{-1} \cdot f_{\alpha \beta}(\xi)$ 
follows. Taking a partition of unity $\left\{j_{\alpha}\right\}$ subordinate to $\left\{U_{\alpha}\right\}$, define $r_{\alpha}: \Omega_{\omega}^{U_{\alpha}} \rightarrow E_{w}$ by

$$
r_{\alpha}(\xi)=\sum_{\beta} j_{\rho}(b \xi) f_{\lambda \beta}(\xi)
$$

and $\bar{n}_{\alpha}: \Omega_{w}^{U} \rightarrow \Phi_{w}^{U_{\alpha}}$ by

$$
\bar{n}_{\alpha}(\xi)=n_{\alpha}(\xi) . \iota\left(r_{\alpha}(\xi)\right)
$$

Since the action is linear,

and therefore

$$
\begin{aligned}
r_{\alpha}(\xi \cdot g) & =\sum_{\beta} j_{\beta}(b \xi) \cdot f_{\alpha \beta}(\xi \cdot g) \\
& =\sum_{\beta} j_{\beta}(b \xi) \cdot g^{-1} \cdot f_{\alpha \beta}(\xi) \\
& =g^{-1} \cdot r_{\alpha}(\xi)
\end{aligned}
$$

$$
\begin{aligned}
\bar{n}_{\alpha}(\xi \cdot g) & =n_{\alpha}(\xi) \cdot n_{w}(g) \cdot \iota\left(r_{\alpha}(\xi \cdot g)\right) \\
& =n_{\alpha}(\xi) \cdot\left(n_{w}(g) \cdot \iota\left(r_{\alpha}(\xi \cdot g) \cdot n_{u}\left(g^{-1}\right)\right) \cdot n_{w}(g)\right. \\
& =\bar{n}_{\alpha}(\xi) \cdot n_{u}(g)
\end{aligned}
$$

so that $\bar{n}_{\alpha}$ is a principal fibre bundle morphism $\Omega_{w}^{T{ }_{\alpha}} \rightarrow \Phi_{w}^{U}$. Clearly $\bar{n}_{\alpha}$ and $\bar{n}_{\beta}$ agree on the intersection of their domains so they globalize to a principal fibre bundle morphism $\bar{n}: \Omega_{w} \rightarrow \Phi_{w}$. It is easily checked that $\pi \circ \bar{n}=\mathrm{id}_{\Omega_{w}}$ and so $\bar{n}$ induces a continuous groupoid morphism $n: \Omega \rightarrow \Phi$ with $\pi \circ n=\mathrm{id}_{\Omega}$.

(7.3) and (7.5) together yield

THEOREM 4. For a locally trivial, locally compact topological groupoid $\Omega$ on base $B$, and a continuous $\Omega$-module $E, \mathscr{V}$ is an isomorphism, and

$$
\operatorname{LocROpext}(\Omega, E)=\operatorname{ROpext}(\Omega, E) .
$$

Thus all locally rigid operator extensions are in fact rigid, and therefore arise from extensions of any given vertex group by a kind of mixing process. One can in principle obtain an explicit description of this process on the cochain level, because since $\mathscr{H}$ preserves injectives, it can be applied to the standard resolution for $A=E_{w}$, namely

$$
0 \longrightarrow A \underset{\lambda^{0}}{\stackrel{\kappa}{\rightleftarrows}} C(G, A) \underset{\lambda^{1}}{\stackrel{d^{0}}{\rightleftarrows}} C\left(G^{2}, A\right) \underset{\lambda^{2}}{\stackrel{d^{1}}{\rightleftarrows}} \ldots \quad\left(G=\Omega_{w}^{w}\right)
$$

to get another resolution for $E$, namely

$$
0 \rightarrow E \cong \mathscr{M}(A) \rightarrow \mathscr{M}(C(G, A)) \rightarrow \mathscr{H}\left(C\left(G^{2}, A\right)\right) \rightarrow \ldots
$$


and then use the basic construction (3.7) together with the identifications (5.2) to get explicit isomorphisms

$$
S_{n}: \mathscr{H}\left(C\left(G^{n+1}, A\right)\right) \rightarrow F^{n}(\Omega, E)
$$

which induce on the cohomology level the isomorphism of Theorem 3. Even for cocycles this is not, of course, a natural identification since the $S_{n}$ involve the partition of unity used to lift the $\lambda^{n}$ (as in (7.2)). It is therefore not likely to be useful in practice and we omit the details.

Consider Theorems 3 and 4. Theorem 3 appears to suggest that the rigid cohomology is too restrictive, despite the naturality of its construction. However, Theorem $\mathbf{4}$ shows that it is in fact the natural cohomology arising from this category of modules, since it classifies the natural class of operator extensions.

In a future paper we will enlarge the category of $\Omega$-modules considered, allowing a wider class of resolutions and thereby obtaining a more satisfying geometric theory. We postpone until then the study of various topics familiar from the cohomology of groups-normalized cochains, the effect of inner automorphisms, a dual homology theory, for example-which could be developed in the present theory.

\section{References}

R. Bott (1975), "Some remarks on continuous cohomology", Proc. Internat. Conf. on Manifolds and Related Topics (University of Tokyo Press).

R. Brown and J. P. L. Hardy (1976), "Topological groupoids I", Mathematische Nachrichten, 71, 273-286.

G. Choquet (1969), Lectures on Analysis, Vols. 1, 2 (Benjamin, New York).

C. Ehresmann (1959), "Catégories topologiques et catégories différentiables", Coll. Géom. diff. Globales, pp. 137-150 (Bruxelles (1958)).

J. P. L. Hardy (1971), “Topological groupoids” (M.A. thesis, University of Wales).

P. J. Higgins (1971), Categories and Groupoids (van Nostrand, Princeton, N.J.).

G. Hochschild and G. D. Mostow (1962), "Cohomology of Lie groups", Illinois J. Math. 6, 367-401.

S. Lang (1966), Rapport sur la Cohomologie des groupes (Benjamin, New York).

S. Lang (1975), Differential Manifolds (Addison-Wesley, Reading, Mass.).

S. MacLane (1963), Homology (Springer-Verlag, New York).

C. C. Moore (1976), "Group extensions and cohomology for locally compact groups III, IV", Trans. Amer. Math. Soc. 221, 1-58.

J. Pradines (1968), "Troisième théorème de Lie pour les groupoides différentiables", Compt. Rend. Acad. Sci. (Paris) 267, A21-A23.

A. K. Seda (1975), "An extension theorem for transformation groupoids", Proc. Royal Irish Acad. 75(A), 255-262.

J. D. Stasheff (1978), Survey article in Bull. Amer. Math. Soc. (to appear).

Department of Mathematics

Monash University

Clayton, Victoria 3168

Australia 